\title{
Diagnostic value of high-frequency color Doppler ultrasonography examination in combination with anti-cyclic citrullinated peptide antibody testing in rheumatoid arthritis patients
}

\author{
MING-YU WANG $^{1 *}$, XIAN-BIN WANG ${ }^{1 *}$, XUE-HUI SUN ${ }^{1}$, FENG-LI LIU $^{2}$ and SHENG-CHUAN HUANG ${ }^{3}$ \\ Departments of ${ }^{1}$ Rheumatology and Immunology, ${ }^{2}$ Rheumatology and Radiology, and ${ }^{3}$ Rheumatology \\ and Ultrasonography, Yantai Yuhuangding Hospital, Yantai, Shandong 264000, P.R. China
}

Received May 16, 2016; Accepted December 13, 2016

DOI: $10.3892 /$ etm.2017.4056

\begin{abstract}
We studied the diagnostic value of high-frequency color Doppler ultrasonography (HCDU) examination in combination with anti-cyclic citrullinated peptide (anti-CCP) antibody testing in rheumatoid arthritis (RA) patients with finger joint damage. From January 2015 to December 2015, 80 patients diagnosed with RA with finger joints damage were enrolled in this study. Patients were examined with HCDU. Serum anti-CCP antibody level was tested using ELISA, and results were compared with the healthy control group. Results obtained by ELISA demonstrated that the positive rate of anti-CCP antibodies was $73.8 \%$ in the study group, and $10 \%$ in the control group. The negative rate was $26.2 \%$ in the study group, and $90 \%$ in the control group. HCDU examination suggested that the predominantly affected joint by bone erosion of RA with finger joint damage was MCP3 (16.7\%), followed by PIP3 (14.1\%), MCP2 (13.5\%) and PIP2 (12.8\%). The slightest affected joint was thumb metacarpophalangeal joint, followed by thumb, little finger metacarpophalangeal joint and proximal interphalangeal joint. The sensitivity of diagnosis of RA with finger joints damage with both HCDU and CCP antibody examination showed a significantly lower level compared with examination with each one of the methods alone, while specificity showed a significantly higher level. Thus, a combination of HCDU examination and anti-CCP antibody testing can be considered useful to improve the early diagnostic rate of RA. HCDU examination is a sensitive, secure, atraumatic and easily-operated diagnostic method for early RA patients with finger joint damage. When combined
\end{abstract}

Correspondence to: Dr Xian-Bin Wang, Department of Rheumatology and Immunology, Yantai Yuhuangding Hospital, 20 Yuhuangding East Road, Yantai, Shandong 264000, P.R. China E-mail: xian_binwang1@163.com

*Contributed equally

Key words: cyclic citrullinated peptide antibody, high-frequency color Doppler ultrasonography, rheumatoid arthritis, finger joint with anti-CCP antibody testing, it will provide a better chance for RA patients, and give them hope for a better treatment and improved prognosis.

\section{Introduction}

Rheumatoid arthritis (RA) is an autoimmune disease, which might induce ankylosis, malformation, even loss of normal joint function (1). Early diagnosis is usually difficult due to atypical clinical features and the negative result of rheumatoid factor testing (2). Thus, the best time period for starting the treatment could be easily missed and as a result, patients could suffer irreversible joints damage leading to permanent disabilities (3). Therefore, a quick and accurate diagnosis of RA has been in the centre of attention $(4,5)$.

In this study, we investigated the diagnostic value of high-frequency color Doppler ultrasonography (HCDU) examination in combination with anti-cyclic citrullinated peptide (anti-CCP) antibody testing in RA patients with finger joint damage.

\section{Materials and methods}

General information. From January to December 2015, 80 patients (with 162 affected joints) who were diagnosed with RA with finger joint damage were enrolled for this study. There were 44 males and 36 females and the age range was from 21 to 68 years (average, $57.2 \pm 3.6$ years). The disease courses ranged from 8 to 22 months (mean disease course, 25.6 \pm 2.2 months). All the patients met the 1987 American clinical diagnostic criteria (6). During the same period, 50 healthy individuals (100 joints) were enrolled in our control group. They were physically examined in the Yantai Yuhuangding Hospital (Shandong, China). There were 30 males and 20 females in the control group, aged 22 to 67 years (average, 56.5 \pm 3.3 years). Aomparison between general information in both groups did not reveal any significant differences.

Methods. HCDU examination and the serum anti-CCP antibody testing by ELISA were conducted. HCDU examinations were conducted as follows: Cross sections and different gestures of the articulationes digitorum manus were scanned 
Table I. Comparison of the HCDU diagnoses for the two groups ( $n=130)$.

\begin{tabular}{lcccccc}
\hline & & \multicolumn{2}{c}{ Cortex of bone } & & \multicolumn{2}{c}{ Medullary substance of bone } \\
\cline { 3 - 4 } Group & No. of arthroses & Continuously smooth & Discontinuously rough & & Not displayed & Tumor-like lesion \\
\hline Control, $\mathrm{n}=50$ & 100 & 100 & 0 & 134 & 100 & 0 \\
Study, $\mathrm{n}=80$ & 162 & 28 & & 32 & 134 \\
\hline
\end{tabular}

HCDU, high-frequency color Doppler ultrasonography.

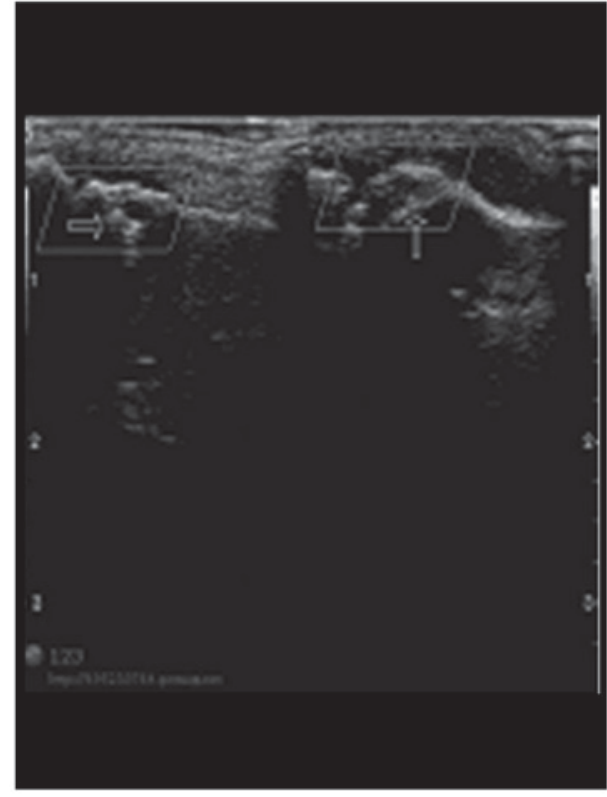

Figure 1. The high-frequency color Doppler ultrasonography manifestation of articulationes interphalangeae of digitus medius of the patients with bone erosion.

with a transducer frequency of $12 \mathrm{MHz}$, using HCDU scanner (Shanghai Chuangxun Medical Equipments Co., Ltd., Shanghai, China). Serum CCP antibody tests using ELISA were conducted by strictly following the instructions provided by the CCP IgG test kit (Beijing Euroimmun Medical Diagnosis Technology Co., Ltd., Beijing, China).

Statistical analysis. SPSS 21.0 (IBM SPSS, Armonk, NY, USA) was used for data analysis. We applied t-test or Chi-square test for comparison between groups. $\mathrm{P}<0.05$ was considered to indicate a statistically significant difference.

\section{Results}

Comparison of the anti-CCP antibody testings. Results obtained from ELISA tests suggested that the positive rate of anti-CCP antibody in the study group was $73.8 \%$ (59/80 cases), and the negative rate was $26.2 \%$ (21/80 cases) (Fig. 1). The positive rate in the control group was only $10 \%$ (5/50 cases), and the negative rate was $90 \%(45 / 50)$.

Comparison of the HCDU examination. HCDU examination results showed that the articulationes interphalangeae of

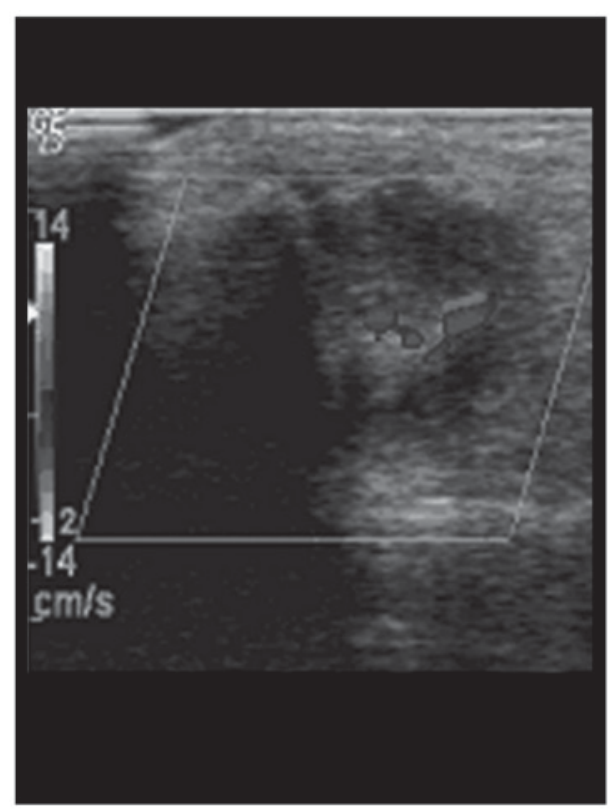

Figure 2. The high-frequency color Doppler ultrasonography manifestation of the apterium blood of the articulationes interphalangeae of digitus medius of the patients with bone erosion

digitus medius in patients with bone erosion in the study group showed articular surface roughness, and continuous interruption at the margins (Fig. 1). The detectable blood signal rate of articulationes interphalangeae in the study group was $65.7 \%$, and blood signals were detected inside the articulationes interphalangeae and at the margins (Fig. 2). The eroded cartilage and subcortex medullary bone substance in the study group had lower echogenicity and irregularly defined tumor-like lesion. The internal echo was uneven (Table I).

No blood signals were detected in the finger joints in the control group, cortical bones and articular surfaces were relatively smooth with continuous integrity. The ultrasonic manifestation of cortical bones and subchondral bones showed a strong echo line with continuous smoothness at the posterior edge of cartilage. Behind that was the attenuation region and the medullary substance of bone that could not be displayed (Fig. 3 and Table I).

Affected levels of bone erosion of 80 patients. HCDU examination results showed that the predominantly affected joint by bone erosion was MCP3 (16.7\%), followed by PIP3 (14.1\%), MCP2 (13.5\%) and PIP2 (12.8\%). The slightest affected joint was the thumb metacarpophalangeal joint, followed by thumb, 
Table II. Result of diagnosing RA with a combination method of $\mathrm{CCP}$ antibody testing and HCDU examination.

\begin{tabular}{lcc}
\hline Method & Sensitivity, \% & Specificity, \% \\
\hline CCP antibody testing & 72.7 & 82.4 \\
HCDU examination & 71.1 & 83.8 \\
Combination of 2 methods & $47.3^{\mathrm{a}, \mathrm{b}}$ & $98.5^{\mathrm{a}, \mathrm{b}}$ \\
\hline
\end{tabular}

Compared with $\mathrm{CCP}$ antibody testing, ${ }^{\mathrm{a}} \mathrm{P}<0.05$; compared with HCDU examination, ${ }^{b} \mathrm{P}<0.05$. RA, rheumatoid arthritis; CCP, cyclic citrullinated peptide; HCDU, high-frequency color Doppler ultrasonography.



Figure 3. The high-frequency color Doppler ultrasonography manifestation of the articulationes interphalangeae of digitus medius of the control group.

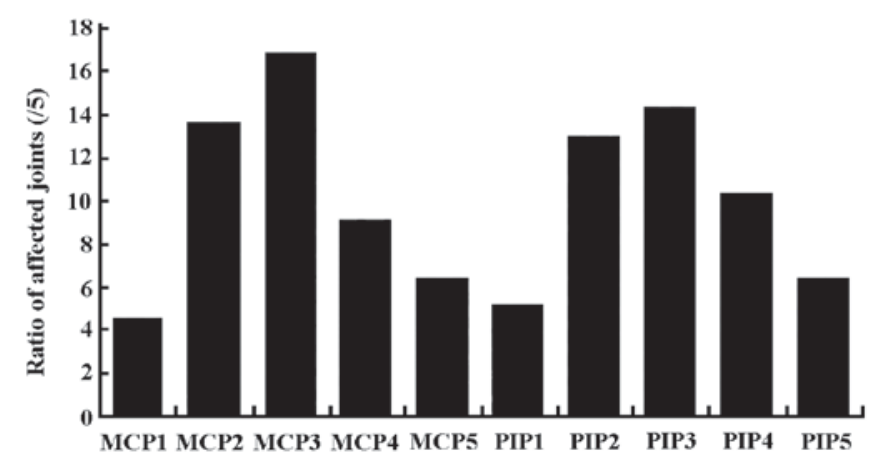

Figure 4. Distribution of affected erosion suggested by high-frequency color Doppler ultrasonography. MCP, thumb metacarpophalangeal joint; PPI, proximal interphalangeal joint; $1-5$, thumb to little finger, respectively.

little finger metacarpophalangeal joint and proximal interphalangeal joint (Fig. 4).

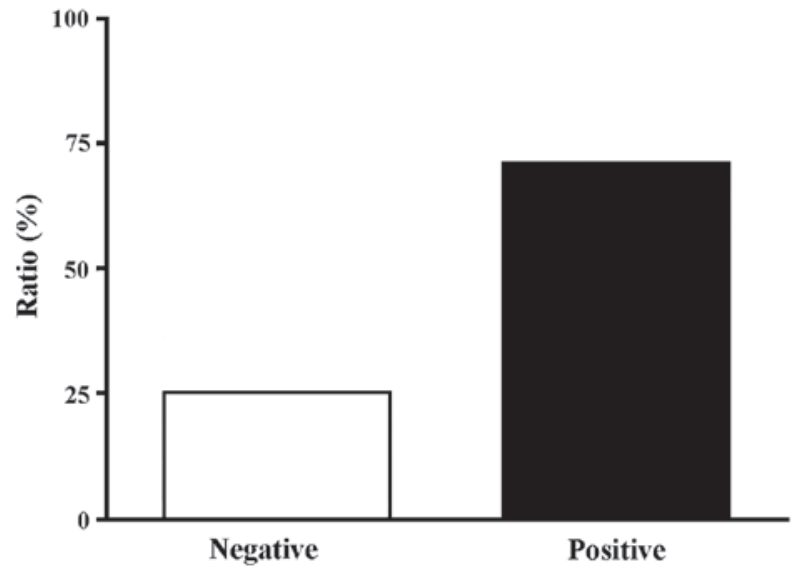

Figure 5.Cyclic citrullinated peptide antibody testing results of the study group.

Result of diagnosing $R A$ with a combination method of $C C P$ antibody testing and HCDU examination. The sensitivity of diagnosing RA with both anti-CCP antibody testing and HCDU examination was obviously lower than using each one of these methods alone $(\mathrm{P}<0.05)$. The specificity was significantly higher $(\mathrm{P}<0.05$; Table II) (Fig. 5).

\section{Discussion}

RA is a chronic inflammatory disorder that characteristically affects the small joints in hands and feet (7). RA generally starts in hand and foot joints progressing to other areas including the knees, hips, and shoulders. The key RA symptom is swollen joints, but RA can also cause fatigue, fever, and weight loss. RA symptoms can cause the joints to become permanently deformed over time which may lead to permanent disability. As there is no cure for this disease, disability caused by RA is irreversible. Prior studies demonstrated that early diagnosis and treatment were effective on the patient condition and lowered the osteoarticular damage and improved prognosis. X-ray, often, is used as a routine method for RA diagnosis; however, depending on the method of X-ray examination, RA could only be detected several years after the appearance of the symptoms $(6,8,9)$.

Blood test to detect rheumatoid factors is also a diagnostic tool which has its limitations (10-12). In recent years, HCDU examination has received significant consideration for indication of early diagnosis in arthritis with no trauma that can be repeatedly operated (13-15). In this study, the HCDU examination was indicated in RA patients suffering from comparative articular surface roughness and continuous interruption at the margins. Blood signals could be observed on inner finger joints and at the margins. The eroded cartilage and subcortex medullary substance of bone had low echogenicity and irregularly defined tumor-like lesion, and the internal echo was uneven. By contrast, HCDU examination on the control group showed no blood signals in the finger joints, cortical bones and articular surfaces were relatively smooth with continuous integrity. Ultrasonic manifestation of cortical bones and subchondral bones had a strong echo line with continuous smoothness at the posterior edge of cartilage. Behind that was the attenuation region and the medullary substance of bone could not be displayed. 
The positive blood ratio in interphalangeal joint detected with HCDU previously (16) was $10 \%$, while the ratio in this study was $65.7 \%$. Our results suggested that by HCDU examination, the predominantly affected joint by bone erosion was MCP3 (16.7\%), followed by PIP3 (14.1\%), MCP2 (13.5\%) and PIP2 (12.8\%). The least affected joint was the thumb metacarpophalangeal joint, followed by thumb, little finger metacarpophalangeal joint and proximal interphalangeal joint. Anti-CCP antibody test is particularly useful in the diagnosis of RA. Anti-CCP antibody is a rheumatoid factor and elevated level of anti-CCP is a sign that the patient may be more likely to develop the disease $(17,18)$. Studies suggested (19) that CCP antigens appeared in the RA patients in early stage, and stimulated the proliferation of $\mathrm{T}$ cells. However, the appearance of the anti-CCP antibodies was highly related to the osteoarticular damage.

A prior study that conducted radiological evaluation of the RA patients with 6 years of follow-up showed that patients who were tested positive for anti-CCP antibody suffered more severe bone damage than those with negative results (20). Other studies suggested that anti-CCP antibody was related to RA bone erosion to a certain degree (21). Results obtained in this study showed that the positive rate of anti-CCP antibody in the study group was $73.8 \%$, and the negative rate was $26.2 \%$ (Fig. 5). The positive rate in the control group was $10 \%$ (and the negative rate was $90 \%$. Reports on the use of HCDU examination in combination with anti-CCP antibody testing in diagnosing RA are rare. Our results suggested that the sensitivity of diagnosing RA with both anti-CCP antibody testing and HCDU examination was obviously lower than using each of those methods alone, while the specificity was significantly higher.

We concluded that a combination of HCDU examination and anti-CCP antibody testing can be considered useful to improve the early diagnostic rate of RA. HCDU examination is a sensitive, secure, atraumatic and easily-operated diagnostic method for early RA patients with finger joint damage. Combined with anti-CCP antibody testing, it will provide a better chance for RA patients, and give them hope for a better treatment and improved prognosis.

\section{References}

1. Botar-Jid C, Bolboaca S, Fodor D, Bocsa C, Tamas MM, Micu M, Dudea SM, Vasilescu D and Badea R: Gray scale and power Doppler ultrasonography in evaluation of early rheumatoid arthritis. Med Ultrason 12: 300-305, 2010.

2. Ceccarelli F, Perricone C, Fabris M, Alessandri C, Iagnocco A, Fabro C, Pontarini E, De Vita S and Valesini G: Transforming growth factor $\beta 869 \mathrm{C} / \mathrm{T}$ and interleukin $6-174 \mathrm{G} / \mathrm{C}$ polymorphisms relate to the severity and progression of bone-erosive damage detected by ultrasound in rheumatoid arthritis. Arthritis Res Ther 13: R111, 2011.

3. Rizzo C, Ceccarelli F, Gattamelata A, Vavala C, Valesini G and Iagnocco A: Ultrasound in rheumatoid arthritis. Med Ultrason 15: 199-208, 2013

4. Hsu HJ, Yang YH, Shieh TY, Chen $\mathrm{CH}$, Kao YH, Yang CF and Ko EC: Role of cytokine gene (interferon- $\gamma$, transforming growth factor- $\beta 1$, tumor necrosis factor- $\alpha$, interleukin- 6 , and interleukin-10) polymorphisms in the risk of oral precancerous lesions in Taiwanese. Kaohsiung J Med Sci 30: 551-558, 2014.
5. Orguc S, Tikiz C, Aslanalp Z and Erbay PD: Comparison of OMERACT-RAMRIS scores and computer-aided dynamic magnetic resonance imaging findings of hand and wrist as a measure of activity in rheumatoid arthritis. Rheumatol Int 33: 1837-1844, 2013.

6. Yang H, Rivoire J, Hoppe M, Srikhum W, Imboden J, Link TM and Li X: Computer-aided and manual quantifications of MRI synovitis, bone marrow edema-like lesions, erosion and cartilage loss in rheumatoid arthritis of the wrist. Skeletal Radiol 44: 539-547, 2015.

7. Tamai M, Kawakami A, Iwamoto N, Kawashiri SY, Fujikawa K, Aramaki T, Kita J, Okada A, Koga T, Arima K, et al: Comparative study of the detection of joint injury in early-stage rheumatoid arthritis by magnetic resonance imaging of the wrist and finger joints and physical examination. Arthritis Care Res (Hoboken) 63: 436-439, 2011.

8. Teruel JR, Burghardt AJ, Rivoire J, Srikhum W, Noworolski SM, Link TM, Imboden JB and Li X: Bone structure and perfusion quantification of bone marrow edema pattern in the wrist of patients with rheumatoid arthritis: A multimodality study. J Rheumatol 41: 1766-1773, 2014.

9. Carter JD, Patelli M, Anderson SR, Prakash N, Rodriquez EJ, Bateman H, Sterrett A, Valeriano J and Ricca LR: An MRI assessment of chronic synovial-based inflammation in gout and its correlation with serum urate levels. Clin Rheumatol 34: 345-351, 2015.

10. Feehan L, Buie H, Li L and McKay H: A customized protocol to assess bone quality in the metacarpal head, metacarpal shaft and distal radius: a high resolution peripheral quantitative computed tomography precision study. BMC Musculoskelet Disord 14: 367, 2013.

11. Bensaoud N, Rostom S, Bahiri R and Hajjaj-Hassouni N: Efficacy of tocilizumab on MRI-determined bone oedema in rheumatoid arthritis. Clin Rheumatol 34: 1031-1037, 2015.

12. Yu JI, Park YR, Lee SS and Chae SC: Polymorphisms of interleukin-31 are associated with anti-CCP levels in females with rheumatoid arthritis. J Genet 93: 813-817, 2014.

13. Yousefghahari B, Alhooei S, Soleimani-Amiri MJ and Guran A: Comparison of sensitivity and specificity of anti-CCP and anti-MCV antibodies in an Iranian cohort of patients with rheumatoid arthritis. Caspian J Intern Med 4: 702-706, 2013.

14. Arkfeld DG: Biological significance of anti-cyclic citrullinated peptide antibody in rheumatoid arthritis. Ann Intern Med 148: 403-404, author reply 403-404, 2008.

15. Alexiou I, Germenis A, Ziogas A, Theodoridou K and Sakkas LI: Diagnostic value of anti-cyclic citrullinated peptide antibodies in Greek patients with rheumatoid arthritis. BMC Musculoskelet Disord 8: 37, 2007.

16. Moghimi J, Ghorbani R, Hasani F and Sheikhvatan M: Discriminative and diagnostic value of anti-cyclic citrullinated peptide antibodies in Iranian patients with rheumatoid arthritis. Rheumatol Int 33: 601-605, 2013.

17. Yang DH, Tu CC, Wang SC, Wei CC and Cheng YW: Circulating anti-cyclic citrullinated peptide antibody in patients with rheumatoid arthritis and chronic obstructive pulmonary disease. Rheumatol Int 34: 971-977, 2014.

18. Perez-Alamino R, Garcia-Valladares I, Cuchacovich R, Iglesias-Gamarra A and Espinoza LR: Are anti-CCP antibodies in psoriatic arthritis patients a biomarker of erosive disease? Rheumatol Int 34: 1211-1216, 2014.

19. Vlad V, Berghea F, Libianu S, Balanescu A, Bojinca V, Constantinescu C, Abobului M, Predeteanu D and Ionescu R: Ultrasound in rheumatoid arthritis: volar versus dorsal synovitis evaluation and scoring. BMC Musculoskelet Disord 12: 124, 2011.

20. Kroot EJ, de Jong BA, van Leeuwen MA, Swinkels H, van den Hoogen FH, van't Hof M, van de Putte LB, van Rijswijk MH, van Venrooij WJ and van Riel PL: The prognostic value of anti-cyclic citrullinated peptide antibody in patients with recent-onset rheumatoid arthritis. Arthritis Rheum 43: 1831-1835, 2000.

21. Bas S, Perneger TV, Seitz M, Tiercy JM, Roux-Lombard P and Guerne PA: Diagnostic tests for rheumatoid arthritis: Comparison of anti-cyclic citrullinated peptide antibodies, anti-keratin antibodies and $\operatorname{IgM}$ rheumatoid factors. Rheumatology (Oxford) 41: 809-814, 2002 . 